\title{
Stage IV Thymoma AJCC v8
}

National Cancer Institute

\section{Source}

National Cancer Institute. Stage IV Thymoma A/CC v8. NCI Thesaurus. Code C136353.

Stage IV includes: IVA: (Any T, N1, M0); (Any T, N0,1, M1a); IVB: (Any T, N2, M0, M1a);

(Any T, Any N, M1b). N0: No regional lymph node metastasis. N1: Metastasis in anterior (perithymic) lymph nodes. N2: Metastasis in deep intrathoracic or cervical lymph nodes. M0: No distant metastasis. M1a: Separate pleural or pericardial nodule(s). M1b:

Pulmonary intraparenchymal nodule or distant organ metastasis. (AJCC 8th ed.) 